\title{
Ecthyma Gangrenosum in Paroxysmal Nocturnal Hemoglobinuria
}

\author{
Constanza Martinez-Mera ${ }^{\mathrm{a}} \quad$ Andrea Montes-Torres $^{\mathrm{a}} \quad$ Carlos Gordillo-Vélez $^{\mathrm{b}}$ \\ Javier Sánchez-Pérez ${ }^{a}$

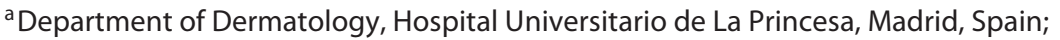 \\ ${ }^{b}$ Department of Pathology, Hospital Universitario de La Princesa, Madrid, Spain
}

A 47-year-old woman presented to the emergency department with a 5-day history of fever and a painful lesion on her left arm. For 25 years she had suffered from paroxysmal nocturnal hemoglobinuria (PNH) with medullary hypoplasia. For the previous 5 years she had been treated with eculizumab $900 \mathrm{mg} / \mathrm{kg}$ every 2 weeks and for the previous year with cyclosporine 3 $\mathrm{mg} / \mathrm{kg} /$ day. Prior to the biological treatment she received meningococcal, pneumococcal, and Haemophilus influenzae vaccines. Serological studies for human immunodeficiency virus, hepatitis $\mathrm{C}$ and $\mathrm{B}$ virus were negative.

The physical examination revealed $38.6^{\circ} \mathrm{C}$ fever and an erythematous, edematous, and painful plaque on the dorsal side of her left arm, showing a violaceous blister of $1.5 \mathrm{~cm}$ at its center (Fig. $1 \mathrm{a}, \mathrm{b}$ ). The lesion was in a different place from the insertion site of the intravenous line that was placed biweekly for eculizumab treatment. There was no adenopathy. Laboratory findings showed hemoglobin $7.9 \mathrm{~g} / \mathrm{dL}$ (normocytic hypochromic anemia) (normal range $[\mathrm{N}]: 12 \pm 16$ ), a white blood cell count of $2 \times 10^{9} / \mathrm{L}\left(\mathrm{N}: 4-10 \times 10^{9}\right)$, neutrophils $1.320 \times 10^{9}\left(\mathrm{~N}: 1.5-8 \times 10^{9}\right)$, lympho- cytes $0.38 \times 10^{9}\left(\mathrm{~N}: 1-4 \times 10^{9}\right)$, and a platelet count of $24 \times 10^{9} / \mathrm{L}(\mathrm{N}: 130-400 \times$ $\left.10^{9} / \mathrm{L}\right)$. The neutropenia, lymphopenia, and thrombocytopenia were previously known. A skin biopsy with culture was performed. The histopathological findings revealed a thin epidermis, an intraepidermic and subepidermic blister, with a diffuse inflammatory cell infiltrate, composed largely of neutrophils, with dermis and subcutaneous necrosis (Fig. 1c). Tissue culture showed the growth of Pseudomonas aeruginosa (PA), which did not grow in repeated blood cultures. The patient was treated empirically with intravenous ceftazidime, amikacin, and teicoplanin in standard doses. After 10 days of intravenous treatment she responded well.

During her hospitalization she maintained her daily treatment with cyclosporine and received her usual doses of eculizumab every 2 weeks ( 3 days before and 1 day after hospitalization). Then she was discharged with ciprofloxacin $750 \mathrm{mg} /$ day orally for 30 days. The infection was completely resolved at the end of treatment.

Ecthyma gangrenosum (EG) is a rare cutaneous lesion caused most commonly by $P A$ and frequently observed in neutro- penic patients. It rarely develops in a $P A$ infected patient in the absence of bacteremia. It is characterized by a gunmetal gray infarcted macule or papule with surrounding erythema, which evolves into a necrotic, black, ulcerative eschar with an erythematous halo. The skin lesions usually appear in the gluteal and perineal regions $(57 \%)$ or extremities $(30 \%)$ but could appear anywhere [1].

An extensive review of EG and EG-like cases, analyzing 167 cases described in the literature from 1975 to 2014, showed that in $26.35 \%$ of cases similar clinical lesions could originate from distinct microorganisms such as Escherichia coli, Klebsiella pneumoniae, Candida albicans, Fusarium, etc. It is described that $58 \%$ of the patients with EG caused by $P A$ suffered from sepsis or bacteremia. It is concluded that EG could also develop as a primary lesion without sepsis and bacteremia, and these patients usually have a better prognosis [1].

The principal health problems in $\mathrm{PNH}$ are related to vein or arterial thrombosis and renal failure. A secondary complication is developing a myelodysplastic syndrome or aplastic anemia [2], which was
KARGER

(c) 2018 S. Karger AG, Basel

E-Mail karger@karger.com

www.karger.com/aha
Constanza Martinez-Mera Dermatology Department Diego de Leon 62 ES-28006 Madrid (Spain)

E-Mail c.martinezmera@gmail.com 

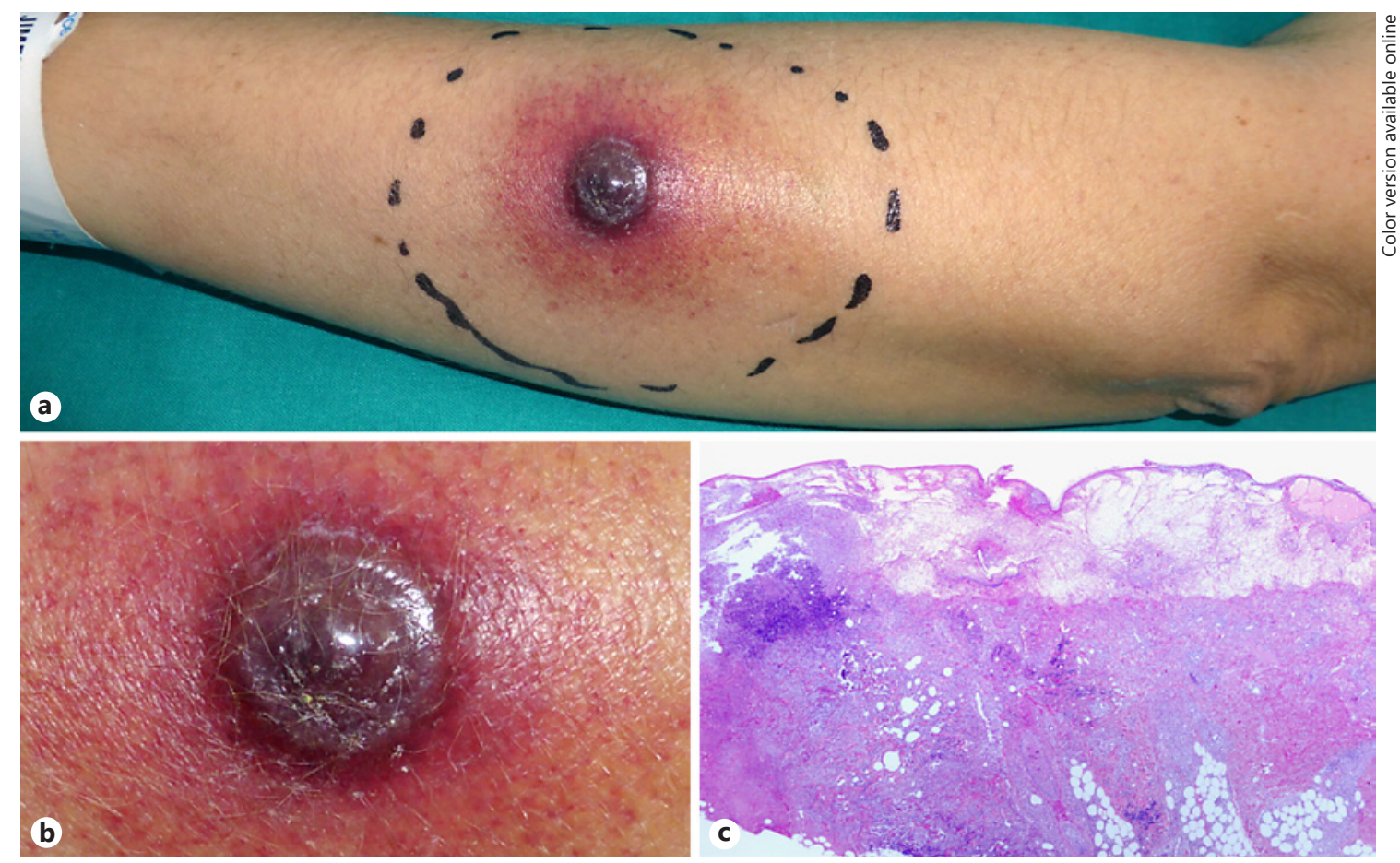

Fig. 1. a Left arm with an erythematous and edematous plaque, with a violaceous blister of $1.5 \mathrm{~cm}$ located in the center. b Amplification of the blister. c Hematoxylin and eosin staining $(\times 5)$ showing a thin epidermis, with intraepidermic and subepidermic blisters and a dense inflammatory infiltrate in the dermis.

not the case in our patient. Cutaneous manifestations in $\mathrm{PNH}$ are rarely reported; they had been described as painful discolored skin lesions when dermal veins are affected, which can ulcerate in rare cases [3]. Purpura fulminans has been reported when the thrombosis compromises an extensive necrotic area [4]. The skin infections in $\mathrm{PNH}$ have been characterized in the literature mainly as necrotizing fasciitis [3].

In 2007, eculizumab, a humanized monoclonal IgG antibody that binds complement protein C5 and inhibits the generation of terminal components of the complement pathway, was approved for the treatment of PNH. This treatment leads to reduced hemolysis, a lower frequency of thromboembolic events, and finally lower transfusion requirements. The Advisory Committee on Immunization Practices (ACIP) recommended immunization against meningococcus in previously unvaccinated individuals starting eculizumab, ideally at least 2 weeks prior to the first dose [5]. Cellulitis and abscess had been reported as uncommon drug side effects.

Some cases of $P A$ with extra cutaneous infection have been reported during treatment with eculizumab. For example, a 34-year-old man, who underwent a haploidentical peripheral blood stem cell transplant for progressive Hodgkin disease complicated by an atypical hemolytic uremic syndrome with recurrent infections with $P A$ during eculizumab therapy, finally died from multiple posttransplant complications including $P A$ pneumonia [6]. A 73-year-old man with $\mathrm{PNH}$ developed $P A$ cholecystitis, liver abscess, and bacteremia during eculizumab therapy, successfully controlled with antibiotics, granulocyte colony-stimulating factor, and cholecystectomy [7].

Cyclosporine is a calcineurin inhibitor which downregulates the activity of T cell immunity, predisposing to mild skin infections like acute folliculitis [8].

The literature suggests a possible underlying pathophysiological association between eculizumab and $P A$ since the complement is known to play an important role in the innate defense against $P A$ and the terminal complement might have a role in the effective killing of these bacteria [6].

Finally, it is possible that the medullary hypoplasia of the patient could predispose her to an immunocompromised state.

To conclude, we herein reported a rare case of PNH treated with eculizumab who developed EG without bacteremia with a good outcome, probably due to early antibiotic administration.

\section{Statement of Ethics}

The patient gave her informed consent for writing the article.

\section{Disclosure Statement}

The authors have no conflicts of interest to declare. The article has no funding sources. 


\section{References}

1 Vaiman M, Lazarovitch T, Heller L, Lotan G. Ecthyma gangrenosum and ecthyma-like lesions: review article. Eur J Clin Microbiol Infect Dis. 2015 Apr;34(4):633-9.

2 Brodsky RA. Paroxysmal nocturnal hemoglobinuria. Blood. 2014 Oct;124(18):2804-11.

3 Patir P, Isik Y, Turk Y, Ugur MC, Ceylan C, Gorgun G, et al. Necrotizing Fasciitis in Paroxysmal Nocturnal Hemoglobinuria. Case Rep Hematol. 2015;2015:908087.
4 Rompoti N, Hillen U, Rösch A, Dissemond J. Purpura fulminans related to paroxysmal nocturnal haemoglobinuria. Eur J Dermatol. 2016 Aug;26(4):397-8.

5 Benamu E, Montoya JG. Infections associated with the use of eculizumab: recommendations for prevention and prophylaxis. Curr Opin Infect Dis. 2016 Aug;29(4):319-29.

6 Webb BJ, Healy R, Child B, Majers J, Anand S, Gouw L. Recurrent infection with Pseudomonas aeruginosa during eculizumab therapy in an allogeneic hematopoietic stem cell transplant recipient. Transpl Infect Dis. 2016 Apr;18(2):312-4
7 Kawakami T, Nakazawa H, Kurasawa Y, Sakai $\mathrm{H}$, Nishina S, Senoo N, et al. Severe Infection of Pseudomonas aeruginosa during Eculizumab Therapy for Paroxysmal Nocturnal Hemoglobinuria. Intern Med. 2018 Jan;57(1): 127-30.

8 Ponticelli C, Bencini PL. Nonneoplastic mucocutaneous lesions in organ transplant recipients. Transpl Int. 2011 Nov;24(11):104150. 